\title{
Neurotoxicity of cerebro-spinal fluid from patients with Parkinson's disease on mesencephalic primary cultures as an in vitro model of dopaminergic neurons
}

\author{
PING KONG ${ }^{1}$, BEN-SHU ZHANG ${ }^{2}$, PING LEI ${ }^{1}$, XIAO-DONG KONG ${ }^{1}$, \\ SHI-SHUANG ZHANG ${ }^{1}$, DAI LI ${ }^{1}$ and YUN ZHANG ${ }^{1}$ \\ Departments of ${ }^{1}$ Geriatrics and ${ }^{2}$ Neurology, Tianjin Medical University General Hospital, Tianjin 300192, P.R. China
}

Received April 21, 2014; Accepted January 29, 2015

DOI: $10.3892 / \mathrm{mmr} .2015 .3575$

\begin{abstract}
Parkinson's disease is a degenerative disorder of the central nervous system. In spite of extensive research, neither the cause nor the mechanisms have been firmly established thus far. One assumption is that certain toxic substances may exist in the cerebro-spinal fluid (CSF) of Parkinson's disease patients. To confirm the neurotoxicity of CSF and study the potential correlation between neurotoxicity and the severity of Parkinson's disease, CSF was added to cultured cells. By observation of cell morphology, changes in the levels of lactate dehydrogenase, the ratio of tyrosine hydroxylase-positive cells, and the expression of tyrosine hydroxylase mRNA and protein, the differences between the two groups were shown. The created in vitro model of dopaminergic neurons using primary culture of mouse embryonic mesencephalic tissue is suitable for the study of neurotoxicity. The observations of the present study indicated that CSF from Parkinson's disease patients contains factors that can cause specific injury to cultured dopaminergic neurons. However, no obvious correlation was found between the neurotoxicity of CSF and the severity of Parkinson's disease.
\end{abstract}

\section{Introduction}

Parkinson's disease is a degenerative disorder of the central nervous system, accounting for $1 \%$ of individuals $>60$ years of age and $4 \%$ of the population $>80$ years (1). Early in the course of the disease, the most obvious symptoms are motoric dysfunctions, including shaking, rigidity, slowness of movement and difficulty with walking (2). As the disease progresses, impaired thinking and behavioral problems may arise,

Correspondence to: Dr Ping Kong, Department of Geriatrics, Tianjin Medical University General Hospital, 154 Anshan Road, Heping, Tianjin 300192, P.R. China

E-mail: pingK1014@163.com

Key words: neurotoxicity, dopaminergic neurons, Parkinson's disease, severity, primary culture whereas depression is the most common psychiatric symptom. However, in spite of extensive research, neither the cause nor the mechanisms underlying the condition have been firmly established thus far. One hypothesis is that certain neurotoxic substances, which may be endogenous or exogenous, may produce excessive amounts of free radicals, which have oxidative properties, eventually leading to the degeneration and the death of dopaminergic neurons (3). Thus, if these types of neurotoxic substances do exist, they may theoretically be present in cerebro-spinal fluid (CSF) from Parkinson's disease patients. Once these neurotoxic substances are identified using current technologies, it will be possible to design available treatments that combat the onset of Parkinson's disease.

Recent studies have found that compounds in CSF from Parkinson's disease patients with a molecular weight $<10 \mathrm{kDa}$ can inhibit the growth of dopaminergic neurons in the midbrain of fetal rats and decrease the number of cultured dopaminergic neurons by $90 \%$. Following incubation with these compounds, the morphology of the surviving dopaminergic neurons was altered, with decreases in size and a lack of extensive connections between dendrites (4). This result suggested that certain neurotoxic substances in CSF of patients with Parkinson's disease may lead to the degeneration of dopaminergic neurons in the substantia nigrapars pars compacta. Another question that arises is whether the composition and amount of neurotoxic substances in CSF from patients with Parkinson's disease vary depending on the level and stage of the disease, which are evaluated by the unified Parkinson's disease rating scale (UPDRS) score and Hoehn \& Yahr classification system (5). In order to investigate this, the present study examined the neurotoxicity of CSF from Parkinson's disease patients on cultured dopaminergic neurons. In addition, the present study attempted to correlate the resulting neurotoxicity with the severity of Parkinson's disease.

\section{Materials and methods}

Patient population and CSF collection. The control group consisted of 32 patients who attended the Surgery or Gynecology department of Tianjin Medical University General Hospital (Tianjin, China) and who were free of diseases of the nervous system. These patients required a lumbar CSF extraction for 
Table I. Demographic data for control and Parkinson's disease patients.

\begin{tabular}{lcccc}
\hline Group & $\mathrm{n}$ & Males, $\mathrm{n}(\%)$ & Females, $\mathrm{n}(\%)$ & Age, years (range) \\
\hline Control & 32 & $19(59)$ & $13(40)$ & $58.7(36-79)$ \\
Parkinson's disease & 32 & $20(62)$ & $12(37)$ & $60.4(46-72)$ \\
\hline
\end{tabular}

diagnostic purposes. The Parkinson's disease group consisted of 32 patients who attended the Neurology department of the General Hospital Affiliated to Tianjin Medical University (Tianjin, China). The demographic characteristics of control and Parkinson's disease patients are summarized in Table I. The clinical diagnosis of probable Parkinson's disease was established considering each patient's medical history, physical examination and laboratory examination and the severity was confirmed according to the criteria recommended by UPDRS and Hoehn \& Yahr (5). The levels of Parkinson's disease in the patients of the present study according to the UPDRS score and Hoehn \& Yahr classification are shown in Table II.

CSF from the control or Parkinson's disease group was taken by routine lumbar puncture following informed consent was obtained from each patient or family members. Following centrifugation at $755 \mathrm{x}$ g for $10 \mathrm{~min}$, the samples of 3-4 ml CSF were aliquoted and stored at $-70^{\circ} \mathrm{C}$ until analysis. Every CSF sample was tested by routine and biochemical examination and the results were in the normal range. The present study was approved by the ethics committee of Tianjin Medical University (Tianjin, China).

Primary culture of dopaminergic neurons. Fetal rats were obtained from pregnant Wistar rats (Medicine Research Institute Animal Center, Military Medical Science Academy of the PLA, Tianjin, China), who were anesthetized by intraperitoneal injection with $3 \mathrm{ml} / \mathrm{kg} 10 \%$ chloral hydrate (Sigma-Aldrich, St. Louis, MO, USA) at embryonic day 14. Under aseptic conditions, the embryonic rostral mesencephalic tegmentum was dissected out from a total of 12-15 embryos according to methods described by Grothe et al (6) and Matarredona et al (7). Each rostral mesencephalic tegmentum (1.0x $1.5 \times 0.75 \mathrm{~mm}$ in size) was further cut into pieces and placed into a sterile petri dish without Hank's balanced salt solution.

The pieces of all rostral mesencephalic tegmentums were mixed together and then transferred to a $10-\mathrm{ml}$ conical plastic tube supplied with $0.25 \%$ tryptase (Sigma-Aldrich) at a volume of 5-10 times the volume of the tissue and digested at room temperature for $30 \mathrm{~min}$. Following digestion, DMEM/F-12 (Gibco-BRL, Invitrogen Life Technologies, Carlsbad, CA, USA) containing $0.04 \mathrm{mg} / \mathrm{ml}$ DNase (Sigma-Aldrich) was added and the mixture was incubated at $37^{\circ} \mathrm{C}$ for $8-10 \mathrm{~min}$, as described in detail previously (8-10). The resulting single cell suspension seeded into a 24 -well cell culture plate pre-coated coated with polylysine and then cultured under standard conditions $\left(37^{\circ} \mathrm{C}, 5 \% \mathrm{CO}_{2}\right)$. After $24 \mathrm{~h}$, the culture medium was changed from DMEM/F-12 to serum-free medium, which was replaced every second or third day. The cultures were grown for 30 days, and images were captured using an Olympus D-00610 microscope (Olympus, Tokyo, Japan).
Addition of CSF to cultured cells. At the 7th day of cell culture, all culture medium was aspirated and replaced with $250 \mu 1$ serum-free medium, followed by culturing for $1 \mathrm{~h}$ $\left(37^{\circ} \mathrm{C}, 5 \% \mathrm{CO}_{2}\right)$.

Subsequently, $50 \mu 1 \mathrm{CSF}$ from the Parkinson's disease group and the control group, respectively, was added into different wells, followed by incubation for $0,24,48$ and $96 \mathrm{~h}$ prior to assessment of neurotoxic parameters.

Determination of the protein abundance of lactate dehydrogenase and immunohistochemical staining. The protein abundance of lactate dehydrogenase in the supernatant of the cultured cells was determined using an LDH kit (Beijing Zhongsheng Bioreagent, Beijing, China) following incubation with CSF for 0, 24, 48 and $96 \mathrm{~h}$, respectively $(11,12)$.

Immunostaining for tyrosine hydroxylase. Prior to immunostaining, cells were fixed with $4 \%$ paraformaldehyde, washed with $0.1 \mathrm{M}$ PBS and endogenous peroxidase activity was blocked with $0.3 \% \mathrm{H}_{2} \mathrm{O}_{2}$ and $10 \%$ methanol. Subsequently, the cells were blocked with normal serum, including $10 \%$ normal goat serum and $0.1 \%$ Triton X-100 PBS. Following incubation with CSF, cells were incubated with tyrosine hydroxylase polyclonal antibody I (diluted concentration, $1: 200 ; 4^{\circ} \mathrm{C}$ overnight; cat. no. 10009413-1), II (diluted concentration, 1:200; room temperature $1 \mathrm{~h}$; cat. no. 10604-1) (Amyjet Scientific Inc, Wuhan, China) or III (avidin and biotin, diluted concentration, 1:50; room temperature $1 \mathrm{~h}$; cat. no. ab90130; Abcam, Cambridge, MA, USA), respectively. Immunohistochemical visualization was performed using the Peroxidase Substrate kit (Sigma-Aldrich) in Dulbecco's phosphate balanced salt solution. Positive staining for tyrosine hydroxylase was indicated by brown cytoplasm and a blue nucleus in the dopaminergic neurons, whereas the cytoplasm of tyrosine hydroxylase-negative cells was colorless.

Measurement of tyrosine hydroxylase mRNA by reverse transcription polymerase chain reaction. Total cellular RNA was extracted from dopaminergic neurons by using a TRIzol kit (Invitrogen Life Technologies). For each experiment, $1 \mu \mathrm{g}$ total cellular RNA was reverse transcribed to cDNA by incubation for $60 \mathrm{~min}$ at $37^{\circ} \mathrm{C}$ with $0.5 \mu 1$ avian myeloblastosis virus reverse transcriptase. Polymerase chain reaction was performed using $0.25 \mu 1$ Taq polymerase and $0.5 \mu 1$ of each primer in a final volume of $50 \mu 1$ containing $10 \mathrm{mM}$ of each deoxynucleotide triphosphate. The primers for tyrosine hydroxylase had the following sequences: Forward, 5'-TCAGAGCAGGATGCCAAG-3' and reverse, 5'-CACCTCGAAGCGCACAA-3'; 355 bp. The $\beta$-actin primer sequences were: Forward, 5'-AGACGGGGTCACCCACAC TGTGCCCATCTA-3' and reverse, 5'-CTAGAAGCATTT 
Table II. Course, UPDRS and Hoehn \& Yahr level of Parkinson's disease patients included in the study.

\begin{tabular}{|c|c|c|c|}
\hline No & $\begin{array}{l}\text { Course } \\
\text { (years) }\end{array}$ & $\begin{array}{l}\text { UPDRS } \\
\text { (score) }\end{array}$ & $\begin{array}{l}\text { Hoehn \& yahr } \\
\text { (level) }\end{array}$ \\
\hline P1 & 5 & 41 & 2.5 \\
\hline P2 & 2 & 45 & 2 \\
\hline P3 & 1 & 54 & 2.5 \\
\hline P4 & 1 & 23 & 1.5 \\
\hline P5 & 3 & 52 & 2.5 \\
\hline P6 & 2 & 31 & 1.5 \\
\hline P7 & 1.5 & 22 & 1.5 \\
\hline P8 & 0.5 & 32 & 1.5 \\
\hline P9 & 1 & 39 & 1.5 \\
\hline P10 & 8 & 56 & 2.5 \\
\hline P11 & 4 & 52 & 2 \\
\hline P12 & 5 & 57 & 2.5 \\
\hline P13 & 3 & 48 & 1.5 \\
\hline P14 & 5 & 58 & 2.5 \\
\hline P15 & 1 & 32 & 1.5 \\
\hline P16 & 2 & 39 & 1.5 \\
\hline P17 & 3 & 47 & 2 \\
\hline P18 & 7 & 59 & 2.5 \\
\hline P19 & 1 & 31 & 1.5 \\
\hline P20 & 2 & 36 & 1.5 \\
\hline $\mathrm{P} 21$ & 3 & 40 & 2 \\
\hline P22 & 4 & 45 & 2 \\
\hline $\mathrm{P} 23$ & 1 & 35 & 1.5 \\
\hline P24 & 4 & 53 & 2.5 \\
\hline $\mathrm{P} 25$ & 2 & 46 & 2.5 \\
\hline P26 & 4 & 66 & 4 \\
\hline P27 & 2 & 45 & 2 \\
\hline P28 & 1 & 36 & 1.5 \\
\hline P29 & 5 & 21 & 1.5 \\
\hline P30 & 3 & 47 & 1.5 \\
\hline P31 & 4 & 62 & 2.5 \\
\hline P32 & 3 & 50 & 2.5 \\
\hline
\end{tabular}

UPDRS, unified Parkinson's disease rating scale.

GCGGTGCACGATGGAGGG-3'; 661 bp. The sequence information used to generate the primers was taken from the literature $(13,14)$. In the presence of tyrosine hydroxylase mRNA, a 355-base pair cDNA fragment was expected to be amplified. Thirty cycles of amplification were performed with denaturation at $94^{\circ} \mathrm{C}$ for $45 \mathrm{sec}$, annealing at $58^{\circ} \mathrm{C}$ for $40 \mathrm{sec}$ and extension at $72^{\circ} \mathrm{C}$ for $40 \mathrm{sec}$. Of each polymerase chain reaction product mixture, $10 \mu 1$ were separated electrophoretically on a $2.5 \%$ polyacrylamide gel (Sigma-Aldrich). After adding the samples into the wells, electrophoresis was performed at $75 \mathrm{~V}$ for $20 \mathrm{~min}$. The results were observed and images were captured using a UV analyzer (Hach Company, Loveland, CO, USA). The Takara DL-2000 DNA marker (Takara Bio Inc., Otsu, Japan) was used as the reference standard for the molecular weight. In the gel imaging analysis, gray band values indicated the tyrosine hydroxylase PCR product and the corresponding $\beta$-actin PCR product. The ratio of the two samples was used to indicate the levels of tyrosine hydroxylase mRNA.

Assessment of tyrosine hydroxylase protein by western blot analysis. Protein extraction was performed with a TRIzol kit (Sigma-Aldrich) according to the manufacturer's instructions. The protein concentration was measured using the method by Addona et al (15). The protein samples were loaded onto separate lanes of an $8 \%$ (for tyrosine hydroxylase) or $10 \%$ (for $\beta$-actin) SDS-polyacrylamide gel, subjected to electrophoresis and transferred to a nitrocellulose membrane (Dingguo Biotechnology Company, Beijing, China). The membrane was blocked with $500 \mathrm{mM} \mathrm{NaCl}$ in $20 \mathrm{mM}$ Tris, pH 7.5 (TBST) (Sigma-Aldrich) containing 5\% skimmed milk and then probed with the primary antibodies (1:1,000 in blocking solution) overnight at $4^{\circ} \mathrm{C}$. The secondary antibody (horseradish peroxidase-conjugated goat anti-mouse immunoglobulin G; 1:2,000 (Zhongshan Goldbridge Biotechnology Company, Beijing, China) in blocking solution was applied for $1 \mathrm{~h}$. Following each incubation, the membrane was thoroughly washed with TBST, then the membrane was treated with chemiluminescence reagent (Dingguo Biotechnology Company, Beijing, China) for visualization of the western blot, which was exposed to a Kodak XOMAT AR film (Kodak, Rochester, NY, USA). Control western blots were prepared by omitting the primary antibodies. The specificity of tyrosine hydroxylase and $\beta$-actin was tested using the gene genius gel documentation and analysis system (Beijing Karaltay Scientific Instruments Co., Ltd., Beijing, China).

Statistical analysis. For statistical analysis, SPSS 18.0 (SPSS, Inc., Chicago, IL, USA) was used. Values are expressed as the mean \pm standard error of the mean. Differences between two factors were analyzed using Student's t-test, one-way analysis of variance or Tukey's test. Statistical methods of correlation and linear regression were also used for data analysis. $\mathrm{P}<0.05$ was considered to indicate a statistically significant difference between values.

\section{Results}

Culture of dopaminergic neurons in vitro. Following inoculation for $2 \mathrm{~h}$, most cells were attached to the bottom of the dish, and cells showed a tadpole-like morphology with circular a shape and 1-2 dendrites. 24 h later, cells were equally distributed and appeared halo. Along with the growth of cells, changes in cell morphology were monitored on days $4,6,9$ and 14. Fig. 1 shows that cells exhibited strong refraction, vigorous growth and network formation among dendrites on days 7-11; this was determined to be the suitable time window for observing changes caused by outer factors. After day 11, the cell size gradually decreased and cells eventually began to die.

Tyrosine hydroxylase immunohistochemical staining is a specific staining method for dopaminergic neurons. Staining was performed on day 11 (Fig. 2); the ratio of cells with positive staining, which was indicated by brown cytoplasm, was $90 \%$. The images of the dopaminergic neurons at 
Table III. Comparison of protein abundance of lactate dehydrogenase in Parkinson's disease group and control group $(\bar{x} \pm \mathrm{s})$.

\begin{tabular}{lcccc}
\hline Group & $0 \mathrm{~h}(\mathrm{U} / \mathrm{l})$ & $24 \mathrm{~h}(\mathrm{U} / \mathrm{l})$ & $48 \mathrm{~h}(\mathrm{U} / \mathrm{l})$ & $96 \mathrm{~h}(\mathrm{U} / \mathrm{l})$ \\
\hline Control & $10.24 \pm 3.56$ & $10.88 \pm 4.04$ & $11.63 \pm 2.47$ & $12.27 \pm 2.99$ \\
Parkinson's disease & $11.51 \pm 3.27$ & $12.01 \pm 3.63$ & $16.70 \pm 3.37$ & $67.92 \pm 6.64^{\mathrm{a}}$
\end{tabular}

${ }^{\mathrm{a}} \mathrm{P}<0.05$, compared with the control group.
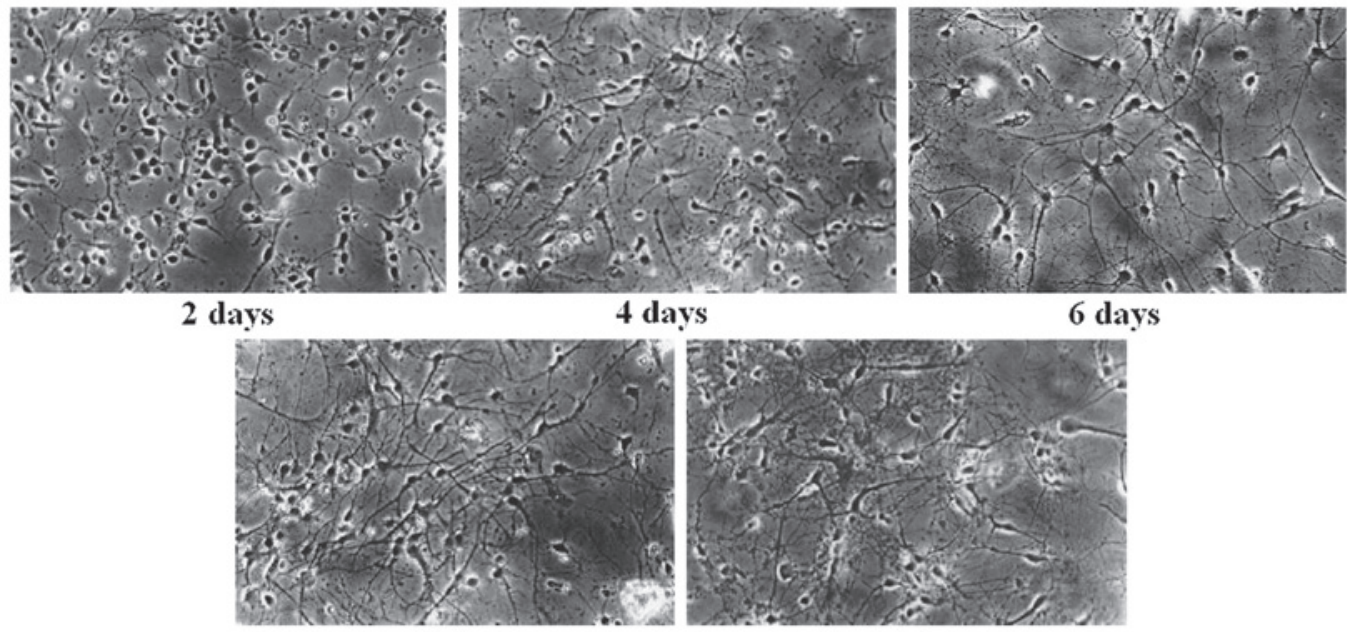

9 days

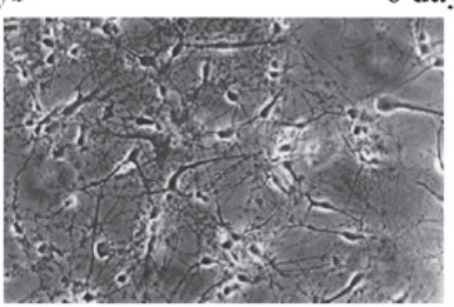

14 days

Figure 1. Morphological changes of mesencephalic cells during the culturing process using immunostaining for tyrosine hydroxylase. Cultured cells were equally distributed and appeared halo after attachment to the dish over $24 \mathrm{~h}$. Along with the growth of cells, morphologies on days $4,6,9$ and 14 are shown (magnification, $\mathrm{x} 400$ ).

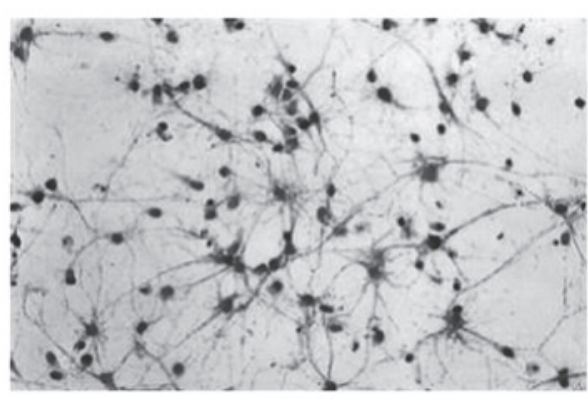

$\times \mathbf{2 0 0}$

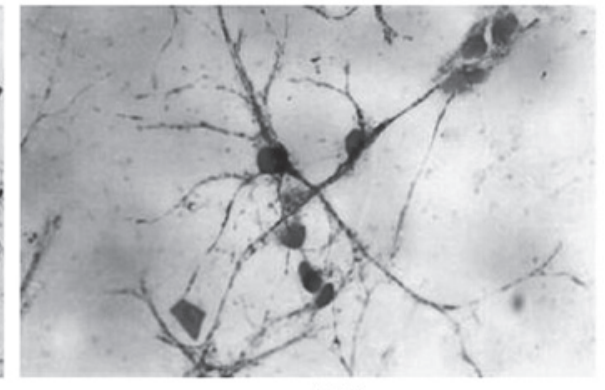

$\times 400$

Figure 2. Immunohistochemical staining for tyrosine hydroxylase in cultured mesencephalic cells on day 11 (magnification, x200 and x400 as indicated).

magnifications of $x 200$ and $x 400$ show their characteristics of triangle-shaped soma, brown cytoplasm and dendrites, round and blue nuclei with round nucleoli as well as connected dendrites (Fig. 2).

CSF from patients with Parkinson's disease causes morphological changes of dopaminergic neurons. Morphological changes were monitored following addition of CSF from patients with Parkinson's disease or the control group for 48 and 96 h (Fig. 3). In the Parkinson's disease group, no obvious decrease in cell size was present at $48 \mathrm{~h}$, however, cell shrinkage appeared at $96 \mathrm{~h}$. Following $96 \mathrm{~h}$ of incubation, the few surviving cells with short and fine dendrites and loss of attachment were observed, and a large number of dendrites emerged around cells. However, in the control group, cells showed a good growth status with elliptic or triangular shapes, obvious halos around the soma as well as pronounced and connected dendrites.

Effect of CSF on cell viability. Following incubation with CSF from the two groups for $0,24,48$ and $96 \mathrm{~h}$, the protein abundance of lactate dehydrogenase was tested (Table III). The protein abundance of lactate dehydrogenase showed gradual increases; however, these were statistically insignificant $(\mathrm{P}>0.05)$. At $96 \mathrm{~h}$, levels of lactate dehydrogenase in the Parkinson's disease group were significantly different from those in the control group $(\mathrm{P}<0.05)$, which was expected, as dead cells were present at this time-point. 
Table IV. Comparison of positive cell ratio (+) in Parkinson's disease group and control group $(\bar{x} \pm \mathrm{s})$.

\begin{tabular}{llr}
\hline Group & $48 \mathrm{~h}(\%)$ & $96 \mathrm{~h}(\%)$ \\
\hline Control & $90.09 \pm 2.19$ & $89.69 \pm 2.07$ \\
Parkinson's disease & $87.53 \pm 2.51$ & $40.53 \pm 3.13^{\mathrm{a}}$ \\
\hline
\end{tabular}

${ }^{\text {aP }}<0.05$, compared with the control group.

Table V. Comparison of relative gray values in Parkinson's disease group and control group $(\bar{x} \pm s)$.

\begin{tabular}{lcr}
\hline Group & $48 \mathrm{~h}(\mathrm{mRNA} / \mathrm{protein})$ & $96 \mathrm{~h}(\mathrm{mRNA} / \mathrm{protein})$ \\
\hline Control & $0.8588 \pm 0.0567 / 0.9760 \pm 0.0409$ & $0.7915 \pm 0.0339 / 0.9640 \pm 0.0503$ \\
Parkinson's disease & $0.8080 \pm 0.0581 / 0.9620 \pm 0.0589$ & $0.5394 \pm 0.0413^{\mathrm{a}} / 0.7400 \pm 0.0431^{\mathrm{a}}$
\end{tabular}

${ }^{\mathrm{a}} \mathrm{P}<0.05$, compared with the control group.
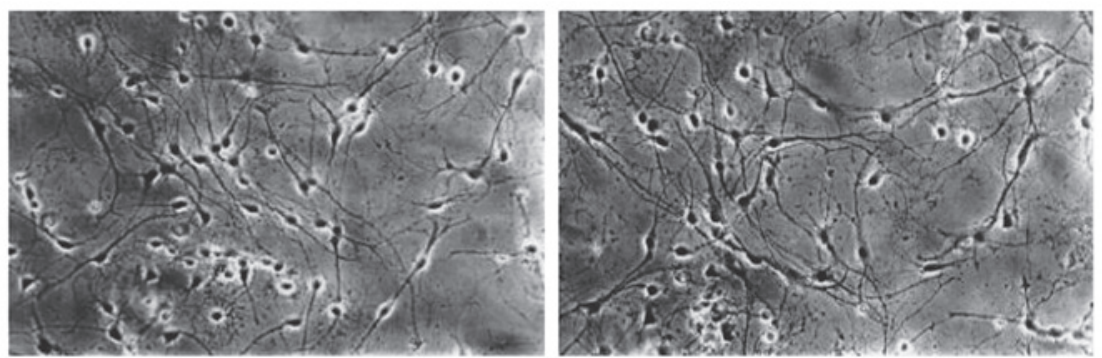

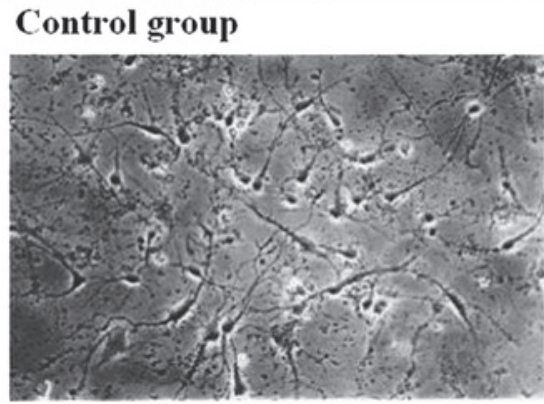

Parkinson's disease Group

$48 \mathrm{~h}$

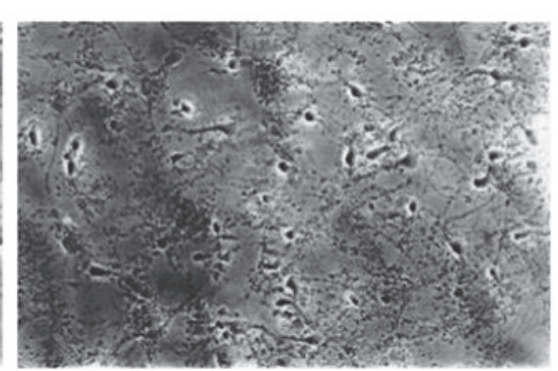

$96 \mathrm{~h}$

Figure 3. Morphological changes in mesencephalic cells following addition of CSF using immunostaining for tyrosine hydroxylase. CSF from patients with Parkinson's disease group and control group were added to cultured mesencephalic cells for 48 and 96 h. CSF, cerebro-spinal fluid (magnification, $\mathrm{x} 400$ ).

Immunohistological examination of tyrosine hydroxylase-positive cells. Following incubation with CSF for 48 and $96 \mathrm{~h}$, tyrosine hydroxylase immunohistochemical staining was performed (Fig. 4, Table IV). No obvious difference between the two groups was observed at $48 \mathrm{~h}(\mathrm{P}>0.05)$ with a tyrosine hydroxylase-positive ratio of $90 \%$. Following $96 \mathrm{~h}$ of incubation, the ratio of tyrosine hydroxylase-positive cells remained unaltered, while in the Parkinson's disease group, the tyrosine hydroxylase-positive cell ratio declined, alongside with the cell number $(\mathrm{P}<0.05)$. It was observed that in the Parkinson's disease group, cells that were tyrosine hydroxylase-positive at $48 \mathrm{~h}$ disappeared at $96 \mathrm{~h}$, while those that were tyrosine hydroxylase-negative at $48 \mathrm{~h}$ remained at $96 \mathrm{~h}$, leading to an overall decline in the tyrosine hydroxylase-positive cell ratio (Fig. 4).
Expression of tyrosine hydroxylase $m R N A$ and protein. Relative gray values of tyrosine hydroxylase/ $\beta$-actin were used to evaluate the expression of tyrosine hydroxylase mRNA and tyrosine hydroxylase protein following incubation with CSF for 48 and $96 \mathrm{~h}$ (Fig. 5, Table V). The results were similar to those obtained by immunohistochemistry; namely that tyrosine hydroxylase levels were significantly decreased in the Parkinson's disease group following $96 \mathrm{~h}$, while remaining unaltered in the other groups and time-points.

Correlation of CSF neurotoxicity with severity of Parkinson's disease. The association between neurotoxicity and severity of Parkinson's disease was analyzed using the increase rate of lactate dehydrogenase, the decrease rate of tyrosine 
Table VI. Analysis of the correlation cerebro-spinal fluid neurotoxicity with the severity of Parkinson's disease.

\begin{tabular}{lccc}
\hline \multirow{2}{*}{ Index } & \multirow{2}{*}{$\begin{array}{c}\text { Course of } \\
\text { Parkinson's disease }\end{array}$} & \multicolumn{2}{c}{ Severity of Parkinson's disease } \\
\cline { 3 - 4 } & $\mathrm{P}=0.768>0.05$ & UPDRS & Hoehn \& Yahr \\
\hline Increase rate of LDH (\%) & $\mathrm{P}=0.079>0.05$ & $\mathrm{P}=0.975>0.05$ & $\mathrm{P}=0.845>0.05$ \\
Loss rate of TH $(\%)$ & $\mathrm{P}=0.682>0.05$ & $\mathrm{P}=0.971>0.05$ \\
\hline
\end{tabular}

UPDRS, unified Parkinson's disease rating scale; LDH, lactate dehydrogenase; TH, tyrosine hydroxylase.
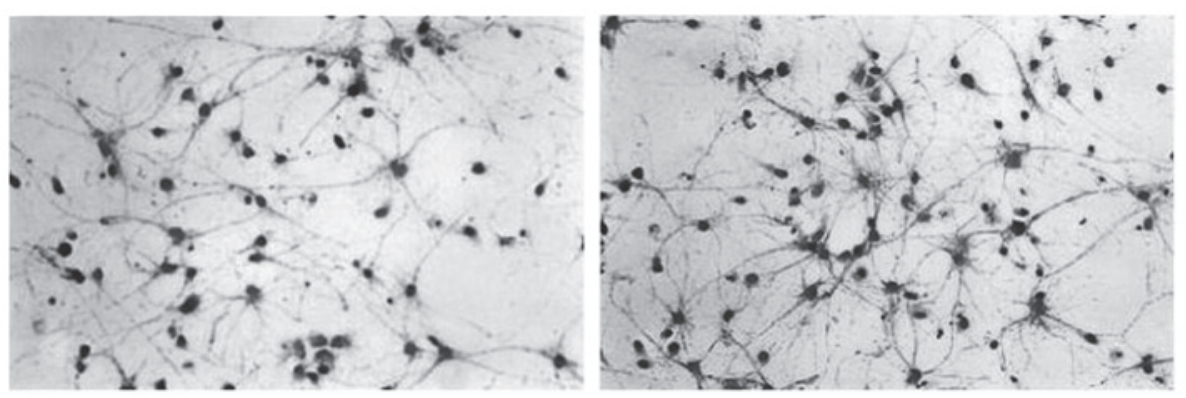

\section{Control Group}

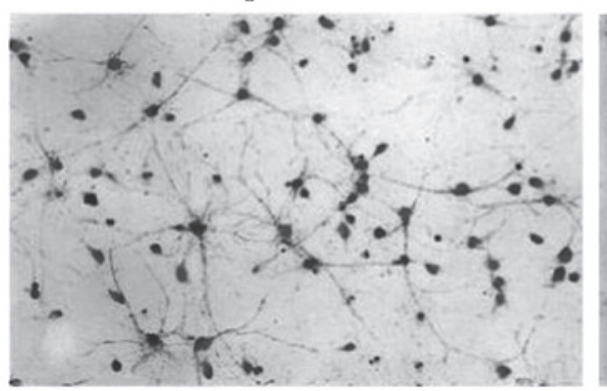

Parkinson's disease Group

$48 \mathrm{~h}$

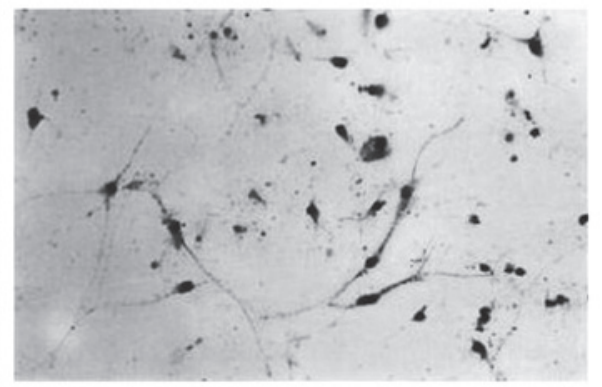

$96 \mathrm{~h}$

Figure 4. Immunohistochemical staining of mesencephalic cells for tyrosine hydroxylase following incubation with cerebro-spinal fluid for 48 and $96 \mathrm{~h}$. (magnification, $\mathrm{x} 200$ ).
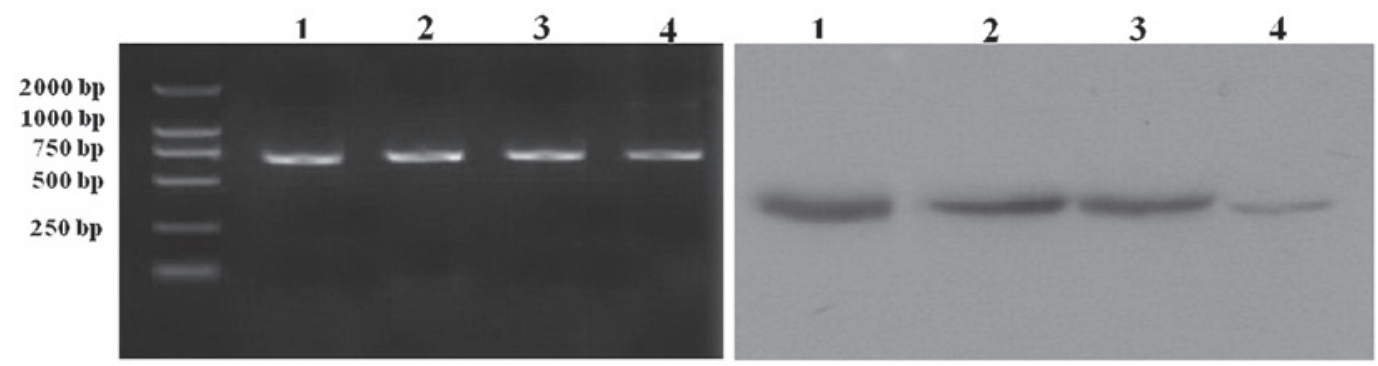

Tyrosine Hydroxylase mRNA

\section{TH Protein}
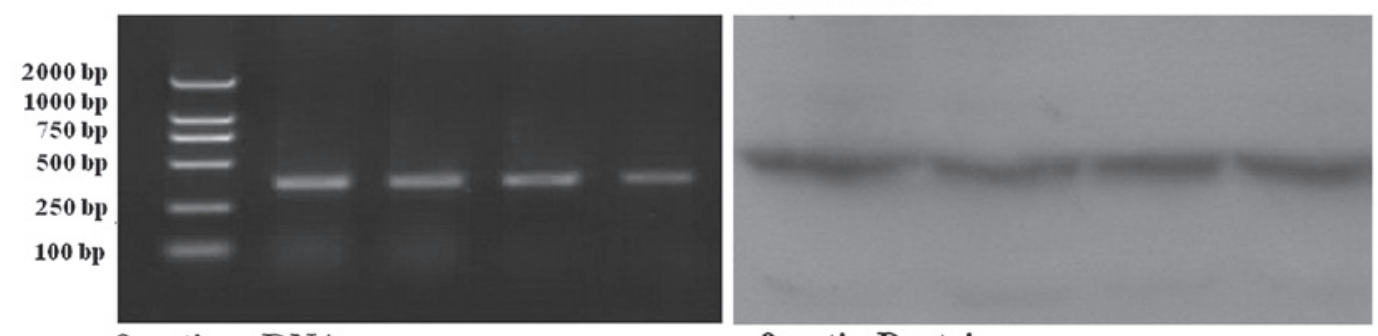

p-actin mRNA

$\beta$-actin Protein

Figure 5. mRNA and protein expression of tyrosine hydroxylase and $\beta$-actin. Lanes 1-2, cells following incubation with CSF from the control group for 48 and $96 \mathrm{~h}$, respectively. Lands 3 and 4, cells following incubation with CSF from Parkinson's disease group for 48 and 96 h, respectively. CSF, cerebro-spinal fluid. 
hydroxylase at $96 \mathrm{~h}$ as well as the UPDRS score and the Hoehn \& Yahr levels (Table VI). The result showed that there was no significant correlation between the UPDRS score or Hoehn \& Yahr classification and the severity of Parkinson's disease $(\mathrm{P}>0.05)$.

\section{Discussion}

Parkinson's disease is a chronic, progressive, neurodegenerative disorder characterized by the loss of dopaminergic neurons in the substantia nigra that innervates the striatum, which has a particularly serious impact on the quality of life of elderly patients (16). As to date, the molecular mechanisms underlying Parkinson's disease have remained to be fully elucidated, studies on the pathogenesis and lesion of the disease are helpful for finding novel treatments.

Most previous studies used live animal models of Parkinson's disease, including monkeys and rats (17). In the present study, in vitro primary culture of mouse embryonic mesencephalic tissue was employed as a model, which avoided variations due to experimental and environmental conditions on the growth of dopaminergic neurons, as these were adjusted on demand. At the same time, cell culture as performed in the present study was characterized by good repeatability, avoiding the numerous complex factors of in vivo experiments, as well as the possibility to directly observe cell morphology and biochemical changes of cells.

The rostral mesencephalic tegmentum of rats at embryonic-day-14 was selected for the generation of primary cells. Previous studies reported that the survival rate of dopaminergic neurons collected at embryonic day 14 was $\sim 17-21 \%$, which was higher than that of cells collected at embryonic day 16 or later, which was only $0.1-2.5 \%(18,19)$. In addition, a previous study confirmed that $\sim 90 \%$ of dopaminergic neurons are clustered around a tissue region of $1.0 \mathrm{~mm}^{3}$ in rostral mesencephalic tegmentum (20). Tyrosine hydroxylase immunohistochemical staining, which is a specific staining method for dopaminergic neurons (21), showed that $\sim 90 \%$ cells were positive at 7-11 days of culture, which were therefore confirmed as being dopaminergic neurons. Day 7 was suitable for adding external factors and changes were noted from days 8-11.

In the present study, four indexes were selected to detect the effect of CSF from patients with Parkinson's disease on dopaminergic neurons; those were cells morphology, changes in lactate dehydrogenase levels, the tyrosine hydroxylase-positive cell ratio by immunohistochemistry staining and the expression of tyrosine hydroxylase mRNA and protein. It is well known that the permeability of the cell membrane is a common feature of cell damage. In live dopaminergic neurons, lactate dehydrogenase is contained within the cell; however, upon membrane perforation, it is released into the cytoplasm. Thereby, lactate dehydrogenase levels in the cell supernatant are a reliable indicator of injury of dopaminergic neurons (22). Furthermore, reverse transcriptase polymerase chain reaction and western blot were also applied in the present study. Reverse transcriptase polymerase chain reaction is characterized by high sensitivity and specificity (23), whereas western blotting combines the high resolution of gel electrophoresis with the specific sensitivity of solid-phase immunoassays (24). By detecting the expression of tyrosine hydroxylase mRNA using reverse transcriptase polymerase chain reaction and tyrosine hydroxylase protein using western blot analysis, differences between the group incubated with CSF from patients with Parkinson's disease and the control group were shown.

The neurotoxicity of CSF from patients with Parkinson's disease was confirmed by the results of the present study. Following $48 \mathrm{~h}$ of incubation with CSF from patients with Parkinson's disease, differences in lactate dehydrogenase levels were observed compared with those the control group; however, these were not significant $(\mathrm{P}>0.05)$. Following $96 \mathrm{~h}$, however, lactate dehydrogenase levels increased by six times those of the control group. Furthermore, at $96 \mathrm{~h}$, the tyrosine hydroxylase-positive cell ratio decreased by $\sim 50 \%$, and the mRNA as well as protein expression of tyrosine hydroxylase decreased $(\mathrm{P}<0.05)$. Following incubation with $\mathrm{CSF}$ from the Parkinson's disease group, the number of tyrosine hydroxylase-positive cells was significantly reduced, while that of tyrosine hydroxylase-negative cells was not significantly changed, which suggested the presence of toxic substances in CSF of Parkinson's disease patients, which may specifically inhibit the growth of dopaminergic neurons in accordance with previous studies $(25,26)$. Although the identity of these toxic substances in CSF of Parkinson's disease patients has yet to be determined, there are numerous theories. One study indicated that biomolecules with a molecular mass $>10 \mathrm{Kda}$ in the CSF were able to promote mesencephalic neuronal survival, while those with a mass $<10 \mathrm{Kda}$ had inhibiting effects (27). Another study reported that thermally stable substances with a mass $<10 \mathrm{Kda}$ possessed neurotoxicity, which indicated that these substances may not be typical proteins $(28,29)$. In 1968, Tanford (30) identified serum albumin, which was able to remain active following denaturation of its tertiary structure due to heating. Consequently, is was thought that peptides without a tertiary structure have a growth-inhibiting effect on dopaminergic neurons. In this light, the results of the present study indicated that CSF from patients with Parkinson's disease, whose components had a wide range of molecular masses, had an inhibiting effect on dopaminergic neurons. Further studies on the neurotoxic activities of molecular components of CSF with different molecular weight ranges are therefore required.

The correlation between neurotoxicity and UPDRS score or Hoehn \& Yahr classification was also studied utilizing the increase rate of lactate dehydrogenase and the decrease rate of tyrosine hydroxylase. Calculations showed that there was no obvious correlation between neurotoxicity and any another factors $(\mathrm{P}>0.05)$. However, owing to the small number of cases, a definitive conclusion cannot be drawn and further studies are required.

\section{Acknowledgements}

The authors would like to thank Professor Peiyu $\mathrm{Pu}$ and Professor Yan Cheng for their support and help with the experiments of the present study. Furthermore, Dr Chunsheng Kang and Professor Guangxiu Wang from the Department of Neurology (Tianjin Medical University, Tianjin, China) are acknowledged for years of guidance and help. 


\section{References}

1. Nichols WC, Pankratz N, Hernandez D, et al; Parkinson Study Group-PROGENI investigators: Genetic screening for a single common LRRK2 mutation in familial Parkinson's disease. The Lancet 365: 410-412, 2005 .

2. Jankovic J: Parkinson's disease: clinical features and diagnosis. J Neurol Neurosurg Psychiatry 79: 368-376, 2008.

3. Przedborski S, Tieu K, Perier C and Vila M: MPTP as a mitochondrial neurotoxic model of Parkinson's disease. J Bioenerg Biomembr 36: 375-379, 2004.

4. Hao R, Norgren RB Jr, Lau YS and Pfeiffer RF: Cerebrospinal fluid of Parkinson's disease patients inhibits the growth and function of dopaminergic neurons in culture. Neurology 45 : $138-142,1995$.

5. Rodriguez-Blazquez C, Rojo-Abuin JM, Alvarez-Sanchez M, et al: The MDS-UPDRS Part II (motor experiences of daily living) resulted useful for assessment of disability in Parkinson's disease. Parkinsonism Relat Disord 19: 889-893, 2013

6. Grothe C, Timmer M, Scholz T, et al: Fibroblast growth factor-20 promotes the differentiation of Nurr1-overexpressing neural stem cells into tyrosine hydroxylase-positive neurons. Neurobiol Dis 17: 163-170, 2004.

7. Matarredona ER, Meyer M, Seiler RW and Widmer HR: CGP 3466 increases survival of cultured fetal dopaminergic neurons Restor Neurol Neurosci 21: 29-37, 2003.

8. Swanson KM, Stelwagen K, Dobson J, et al: Transcriptome profiling of Streptococcus uberis-induced mastitis reveals fundamental differences between immune gene expression in the mammary gland and in a primary cell culture model. J Dairy Sci 92: 117-129, 2009.

9. Huang H, Lindgren A, Wu X, Liu N and Lin S: High-throughput screening for bioactive molecules using primary cell culture of transgenic zebrafish embryos. Cell Reports 2: 695-704, 2012.

10. Kim J, Su SC, Wang H, et al: Functional integration of dopaminergic neurons directly converted from mouse fibroblasts. Cell Stem Cell 9: 413-419, 2011.

11. Nakamura K, Wright DA, et al: Preferential resistance of dopaminergic neurons to the toxicity of glutathione depletion is independent of cellular glutathione peroxidase and is mediated by tetrahydrobiopterin. J Neurochem 74: 2305-2314, 2000.

12. Anderson M: Glutathione: an overview of biosynthesis and modulation. Chem Biol Interact 111-112: 1-14, 1998.

13. Grima B, Lamouroux A, Blanot F, Biguet NF and Mallet J: Complete coding sequence of rat tyrosine hydroxylase mRNA Proc Nat Acad Sci USA 82: 617-621, 1985.

14. Nudel U, Zakut R, Shani M, Neuman S, Levy Z and Yaffe D: The nucleotide sequence of the rat cytoplasmic beta-actin gene. Nucleic Acids Res 11: 1759-1771, 1983.

15. Addona TA, Abbatiello SE, Schilling B, et al: Multi-site assessment of the precision and reproducibility of multiple reaction monitoring-based measurements of proteins in plasma. Nat Biotechnol 27: 633-641, 2009.
16. Santangelo, G, Trojano, L, Barone P, Errico D, Grossi D and Vitale C: Apathy in Parkinson's disease: diagnosis, neuropsychological correlates, pathophysiology and treatment. Behav Neurol 27: 501-513, 2013.

17. Dehay B and Bezard E: New animal models of Parkinson's disease. Mov Disord 26: 1198-1205, 2011.

18. Ma A, Wang L, Gao Y, et al: Tsc1 deficiency-mediated mTOR hyperactivation in vascular endothelial cells causes angiogenesis defects and embryonic lethality. Hum Mol Genet 23: 693-705, 2014.

19. Duan D, Fu Y, Paxinos G and Watson C: Spatiotemporal expression patterns of Pax6 in the brain of embryonic, newborn and adult mice. Brain Struct and Funct 218: 353-372, 2013.

20. Shimoda K, Sauve Y, Marini A, Schwartz JP and Commissiong JW: A high percentage yield of tyrosine hydroxylase-positive cells from rat E14 mesencephalic cell culture. Brain Res 586: 319-331, 1992.

21. Kato Y, Peled N, Wynes MW, et al: Novel epidermal growth factor receptor mutation specific antibodies for non-small cell lung cancer: Immunohistochemistry as a possible screening method for epidermal growth factor receptor mutations. J Thorac Oncol 5: 1551-1558, 2010.

22. Bancos S, Tsai DH, Hackley V, Weaver JL and Tyner KM: Evaluation of viability and proliferation profiles on macrophages treated with silica nanoparticles in vitro via plate-based, flow cytometry and coulter counter assays. ISRN Nanotechnol 2012: 454072, 2012.

23. Hue KD, Tuan TV, Thi HT, et al: Validation of an internally controlled one-step real-time multiplex RT-PCR assay for the detection and quantitation of dengue virus RNA in plasma. J Virol Methods 177: 168-173, 2011.

24. Holste A, Tholey A, Hung C and Schaumlöffel D: Nano-high-performance liquid chromatography with online precleaning coupled to inductively coupled plasma mass spectrometry for the analysis of lanthanide-labeled peptides in tryptic protein digests. Anal Chem 85: 3064-3070, 2013

25. Wasik A, Romańska I and Antkiewicz-Michaluk L: 1-Benzyl-1,2,3,4-tetrahydroisoquinoline, an endogenous Parkinsonism-inducing toxin, strongly potentiates MAO-dependent dopamine oxidation and impairs dopamine release: ex vivo and in vivo neurochemical studies. Neurotox Res 15: 15-23, 2009

26. Mochizuki H: Adult neurogenesis in Parkinson's disease. In: Neurogenesis in the adult brain II: Clinical Implications. Seiki T, Sawamoto K, Parent JM and Alvarez-Buylla A (eds), Springer, Japan, pp23-36, 2011.

27. Yu SJ,Lo ES, Cochran EJ, et al: Cerebrospinal fluid from patients with Parkinson's disease alters the survival of dopamine neurons in mesencephalic culture. Exp Neurol 126: 15-24, 1994.

28. Sanchez-Ruiz JM: Protein kinetic stability. Biophys Chem 148: $1-15,2010$.

29. Mann JP,McCluskey A and Atkin R: Activity and thermal stability of lysozyme in alkylammonium formate ionic liquids-influence of cation modification. Green Chem 11: 785-792, 2009.

30. Tanford C: Protein denaturation. Adv Protein Chem 23: 121-282, 1968. 